\title{
Simulation of diffuse reflectance for characterisation of particle suspensions
}

\author{
Kelly Thomson ${ }^{\mathrm{a}}$, Daria Stoliarskaia ${ }^{\mathrm{a}}$, Sarra Tiernan-Vandermotten ${ }^{\mathrm{a}}$, Leo Lue ${ }^{\mathrm{a}}$, and Yi-Chieh \\ Chen $^{\mathrm{a}}$ \\ ${ }^{a}$ Department of Chemical and Process Engineering, University of Strathclyde, James Weir \\ Building, 75 Montrose St, Glasgow G1 1XJ, UK
}

\begin{abstract}
We employ a physical theory to construct a computational model that accounts for both multiple scattering and absorption of light. The approach does not require a calibration model. Mie theory to describe single particle scattering, which is combined with the diffuse approximation to the radiative transfer equation to provides an analytical prediction of the reflectance. This approach is applied to experimental reflectance measurements on polystyrene particle suspensions with a wide range of particle radii and volume fractions. The method provides good estimates of the suspension properties from a full NIR-vis-UV spectrum.
\end{abstract}

Keywords: diffuse reflectance, radiative transfer theory, diffuse approximation

\section{INTRODUCTION}

Spectroscopy is commonly used to obtain information about the chemical composition of a material; however, for suspensions with a high weight percent of solid, absorption or transmission spectra are difficult to interpret, due to the multiple light scattering by the particulates. It is difficult to distinguish between the contributions of scattering and absorption to the measured spectrum. Theoretical methods are required to disentangle these two processes.

When the concentration of particulates within a sample becomes large, then traditional absorption or transmission spectroscopy will fail. This is due to losses through both scattering and absorption in the medium and the inability to distinguish these two signals. There are many empirical and semi-empirical approaches to correct the scattering induced spectral change, or use physical theory to decouple the absorption and scattering effect. These methods are followed by multivariate analysis to build a calibration model. However, the accuracy and robustness of the model can vary greatly; also, it may not be reliable for another system.

In this article, we implement the diffuse approximation for normal incidence using the method developed by Reynolds et al. together with Mie theory for a series of polystyrene samples with varying radii and concentrations to determined both the radius and the volume fraction after fitting to the reflectance curve.

\section{EXPERIMENTAL AND COMPUTATIONAL METHODS}

\subsection{Experimental details}

The diffuse reflectance measurements were taken experimentally so we could validate our computational model. These measurements consisted of inserting a probe with a light source and receiving fibers. The layout is shown in Fig. 1(a), into a polystyrene suspension sample and switching on the the central source. The backscattered, diffuse reflected, light is then collected on the receiving fibers around the probe and the information is stored in a database. This was repeated for 45 polystyrene suspensions of varying particle concentration and radii. For the full details of the experimental method, see the recent paper by Chen et al ${ }^{1}$

Further author information: (Send correspondence to K.T.)

K.T.: E-mail: kelly.thomson@strath.ac.uk

L.L.: E-mail: leo.lue@strath.ac.uk, Telephone: +44 (0)141 5482470

Y.-C.C.: E-mail: yichieh.chen@strath.ac.uk, Telephone: +44 (0)141548 5304 


\subsection{Diffuse approximation}

The transport of photons through such a material can be described using the radiative transport equation (RTE) ${ }^{2}$ By using this the appropriate absorption and scattering signals can be determined. While it is necessary to use numerical methods to fully solve the RTE ${ }^{3 / 5}$ it can be beneficial to instead use the diffuse approximation to reduce the computational time it takes to run the calculations. The diffuse approximation is limited to systems where the reduced coefficients are much larger than their absorption coefficients and also where the minimum layer thickness is of the order of a few transport mean free paths.

In order to perform this calculation some parameters are required: the absorption coefficient $\mu_{a}$ and the reduced scattering coefficient $\mu_{s}^{\prime}$. The absorption coefficient tells us the chemical composition of the sample and physically represents how far a photon can travel into the sample before it is likely to be absorbed. The reduced scattering coefficient $\mu_{s}^{\prime}$ contains information on the particle size.

The way in which light travels through a colloidal suspension is described by the steady-state radiative transfer equation

$$
\hat{\mathbf{s}} \cdot \nabla I(\mathbf{r}, \hat{\mathbf{s}})=-\mu_{a} I(\mathbf{r}, \hat{\mathbf{s}})-\mu_{s} I(\mathbf{r}, \hat{\mathbf{s}})+\frac{\mu_{s}}{4 \pi} \int d \hat{\mathbf{s}}^{\prime} P\left(\hat{\mathbf{s}}, \hat{\mathbf{s}}^{\prime}\right) I\left(\mathbf{r}, \hat{\mathbf{s}}^{\prime}\right)+S(\mathbf{r}, \hat{\mathbf{s}})
$$

The left side shows the convection flow of photons through the sample and the first term on the right hand side is the absorption term which indicates how far into the sample a photon can travel before it is likely to be absorbed. The next term is the scattering term, this describes how the photons will be scattered through all possible directions within a sphere in space. The final term is the source, here we use a basic exponentially decaying source.$^{6}$ Assuming that the radiance $I(\mathbf{r}, \hat{\mathbf{s}})$ is only sightly anisotropic, it can be approximated in the form

$$
I(\mathbf{r}, \hat{\mathbf{s}}) \approx \frac{1}{4 \pi} \Phi(\mathbf{r})+\frac{3}{4 \pi} \hat{\mathbf{s}} \cdot \mathbf{J}(\mathbf{r})
$$

where $\Phi(\mathbf{r})$ is the local fluence, and $\mathbf{J}(\mathbf{r})$ is local the photon flux. This can be substituted back into Eq. (1) and rearranged to give the diffuse approximation;

$$
\nabla^{2} \Phi(\mathbf{r}, \hat{s})-\frac{\mu_{a}}{D} I(\mathbf{r}, \hat{\mathbf{s}})=\left(1+3 D \mu_{t} g\right) S_{0}(\mathbf{r})
$$

where $D=\left[3\left(\mu_{a}+\mu_{s}^{\prime}\right)\right]^{-1}$, and $S_{0}(\mathbf{r})$ is the source term integrated over all directions.
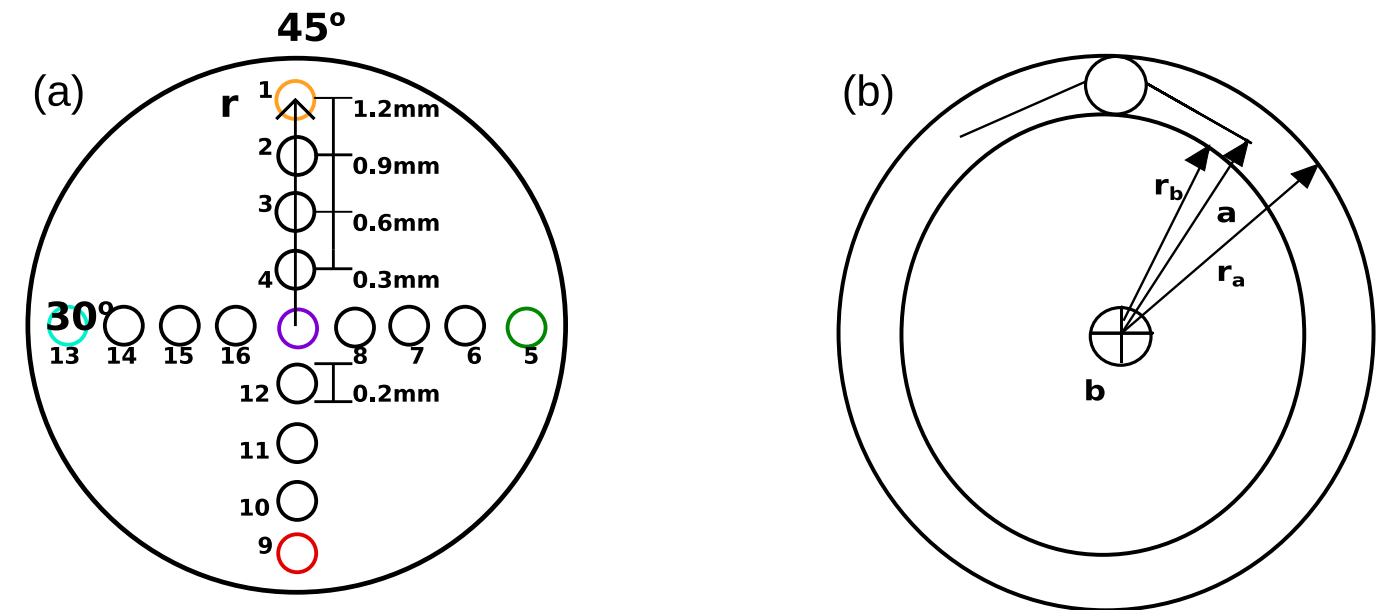

Figure 1. (a) Schematic of the probe layout with the source in the center. (b) Illustration showing the distances from the source. 


\subsection{Mie Theory}

Mie theory is used to estimate both the reduced scattering coefficient and the absorption coefficient for a spectrum ranging from NIR-UV-vis. Mie theory gives the solution of Maxwell's equation for the scattering of a plane wave of light by a spherical particle. In particular, it provides the absorption $\sigma_{a b s}$ and scattering $\sigma_{s c a}$ cross-sections ${ }^{7}$ The required inputs are the diameter of the particle, its complex index of refraction, and the index of refraction of the background medium.

The absorption coefficient $\mu_{a}$ is composed of a contribution from the medium ${ }^{8}$ and the particles.

$$
\mu_{a}=c \sigma_{a b s}+\frac{4 \pi n_{i m}}{\lambda} \phi_{w a t e r}
$$

where $n_{i m}$ is the imaginary part of the refractive index for water, and $c$ is the particle number concentration.

The reduced scattering coefficient $\mu_{s}^{\prime}$ is given by ${ }^{7}$

$$
\mu_{s}^{\prime}=c \sigma_{s c a}(1-g)
$$

where $g$ quantifies the anisotropy of the scattering, which is determined from Mie theory.

\subsection{Inversion procedure}

From an estimate of the particle radius and volume fraction in the suspension, the absorption coefficient and the reduced scattering coefficient can be predicted using Mie theory. With this information, the diffuse approximation ${ }^{6}$ with a sample depth of $100 \mathrm{~mm}$ is then used to compute the reflectance. The square deviation between the predicted and measured diffuse reflectance at $1.2 \mathrm{~mm}$ from the source is minimized by varying the particle radius and concentration using LMFIT $^{[9}$ with the differential evolution method.

\section{RESULTS AND DISCUSSION}

To benchmark our inversion procedure, we first fit to a simulated reflectance spectrum as theory being used to produce this spectrum is the same as ours we should be able to get an exact match.

The initial guess for the volume fraction of the polystyrene particles is 0.05 and a particle radius of $158 \mathrm{~nm}$. The optimizer is run for all the experimental samples, and the predictions for the radius and volume fraction are then compared with diffuse reflectance measurements from polystyrene particle suspensions. The fit method used here is differential evolution, this method attempts to find the global minimum of the system by adjusting the input parameters until the error across the system is as small as it will get.

As a result of experimental conditions the theoretical reflectance which is obtained is of a much smaller value than that of the collected diffuse reflectance, as such a scale factor must be considered. To check that the scale factor is sensible, we first tested the optimizer by only allowing the scale to vary and having the absorption and reduced scattering coefficients read in from the exact experimental radii and concentrations. It was found that the average scale factor was 74.64 and that the standard deviation from this was 10.19 .

We present results of the inversion procedure for the experimental sample with $12.6 \mathrm{wt} \%$ polystyrene particles with a radius of $101.83 \mathrm{~nm}$. We fixed the scale factor to the average found over the experimental samples. The radius was determined to be $87.1 \mathrm{~nm}$ and the volume fraction was 0.123 , and from the fitting procedure, $\chi^{2} \approx 3$. As can be seen from Fig. 2(a), the fit line (dark blue) is in good agreement with what the fibers received during the experiment; however, the fit produces a peak at a wavelength of around $850 \mathrm{~nm}$ corresponding to the absorption behavior shown in Fig. 2(b), while the experimental data does not. This behavior is even seen in our simulation using the exact experimental parameters for the radius and the volume fraction. The reason that this is not seen in the experimental data could be due to peak shifting. However, another explanation for this behavior in the experiment is that it could be due to polydispersity, as we assume in the theory used for the optimizer that we have a monodisperse system. Upon investigation of this possibility it was found that the sample had a polydisperse index of $25.678 \%$ so this could indeed explain the absence of the peak.

Next, we fit the experimental data for this sample again, but this time allowing the scale factor to vary to see. As can be seen in Fig. 3(a), the fit here is worse than before with the behavior at the UV end of the 

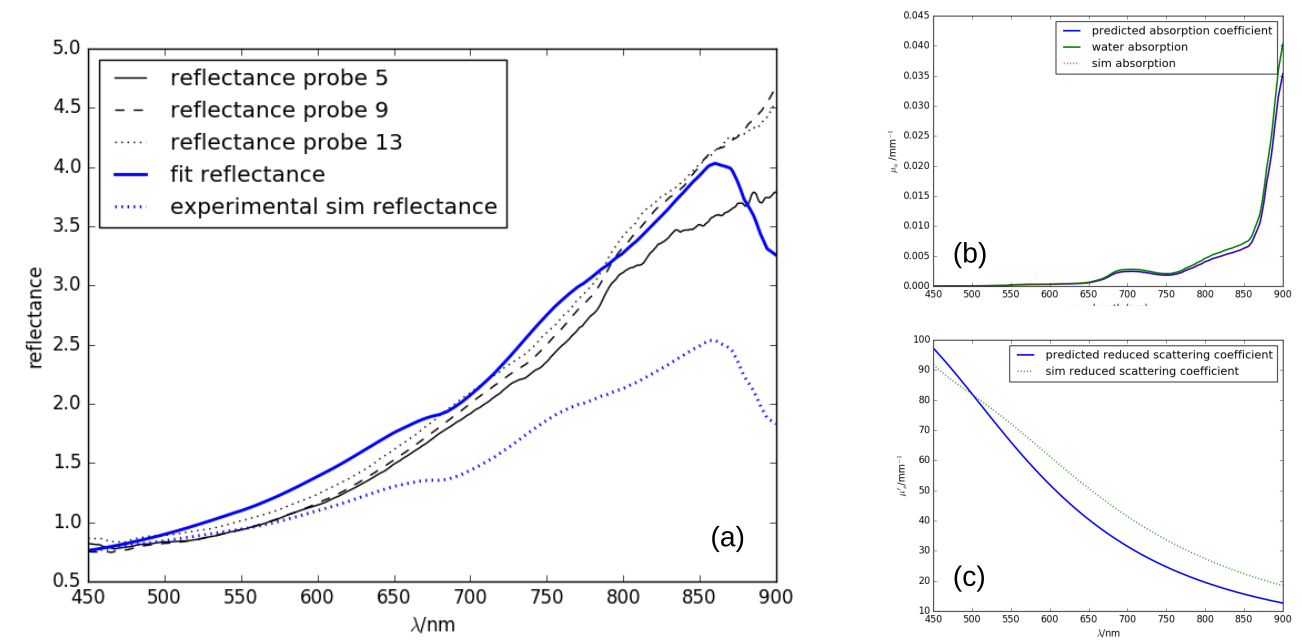

Figure 2. Results of fitting with the scale factor fixed to average value for (a) reflectance, (b) $\mu_{a}$, and (c) $\mu_{s}^{\prime}$.
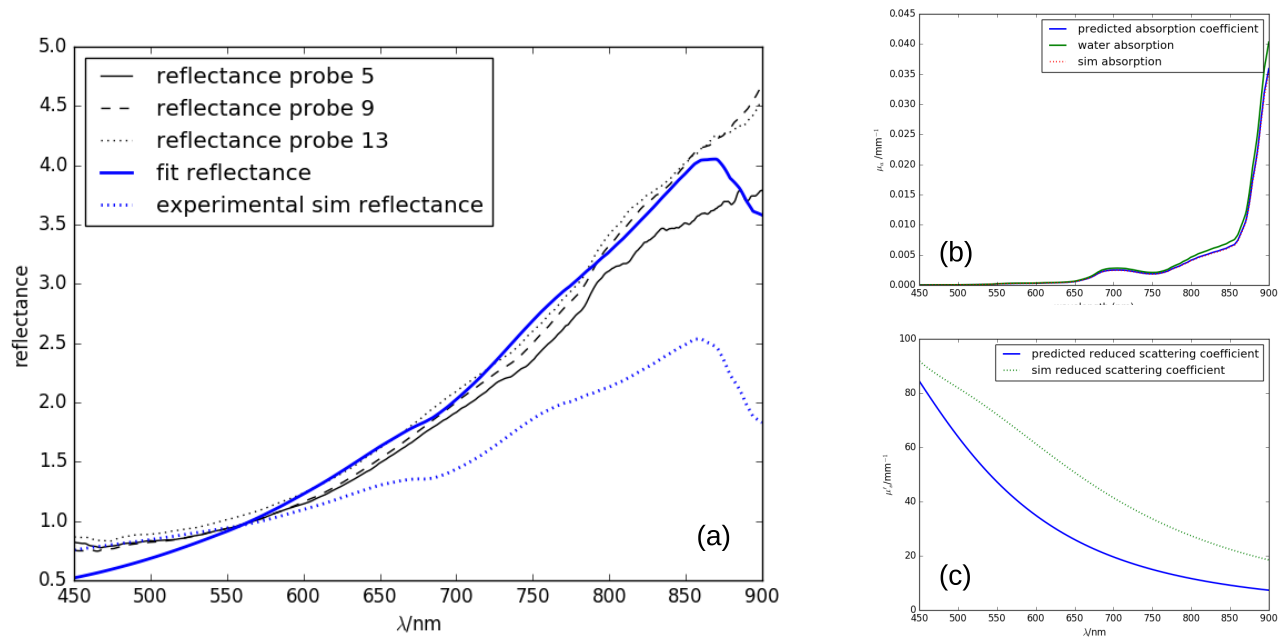

Figure 3. Results of fitting model to experimental reflectance data at two distances allowing the scale factor to vary and for (a) reflectance, (b) $\mu_{a}$, and (c) $\mu_{s}^{\prime}$.

spectrum no longer being captured, this is also reflected in Figs. 3(b) and (c). The predictions found here were that the scale factor should be 44.29 , the radius $73.4 \mathrm{~nm}$ and the volume fraction 0.1093 . When this is compared to experimental values this is quite clearly worse, and this is reflected in the $\chi^{2}$ value as well with it increasing to around 300, an increase by a factor of 100 .

\section{CONCLUSIONS}

In this work, we have developed an inversion procedure which uses the differential evolution method to fit to the diffuse reflectance measurements and produces the radius and volume fraction of polystyrene suspensions by using Mie theory and the diffuse approximation developed by Reynolds et al.$^{6}$ Due to the way in which the experiments are performed, ${ }^{11}$ we had to consider a scale factor in addition to the radius and volume fraction parameters required for the theory used in our optimizer. In order to choose the correct scale factor we used the exact radius and volume fraction measured by the experimentalists ${ }^{1}$ for each of the 45 polystyrene suspensions samples with only the scale factor being allowed to vary. Upon completion of this the average scale factor was 
found to be 74.64 with a standard deviation of 10.19. This meant that the deviation away from this average was was not too significant. It was then decided to run more detailed analysis on a single sample. The optimizer was then run for a scale factor fixed to the average and with the scale factor allowed to vary. It was found that when the scale factor was allowed to vary that the $\chi^{2}$ value increased by a factor of 100 . The predicted radius and the volume fraction produced by the optimizer also became more inaccurate when the scale factor was allowed to vary from a radius of $87.1 \mathrm{~nm}$ to $73.4 \mathrm{~nm}$ with the experimental radius being measured at $101.83 \mathrm{~nm}$ and the volume fraction of 0.123 to 0.1093 with the experimental weight percentage being measured at $12.6 \%$.

In future, we intend to explore the diffuse approximation at angles other than normal incidence and compare

these to experimental values as we have done here. Pioneering work was done by Groenhuis et al. in taking the first steps to provide a closed form expression for the radial variation of diffuse reflectance 10 [11 Groenhuis et al. also developed an approach based on diffusion theory extended to include anisotropic scattering 10 They found that the reflection formula in which the scattering and absorption are described by one parameter each. Monte Carlo simulations were then performed and this showed that the computationally fast approach can be applied 10 This group also developed a new method to determine the absorption and scattering characteristics which involved using the reflection measurement only on the bulk material 11 The absorption and scattering characteristics are usually determined from both the reflection and transmission measurements ${ }^{12}$ Further work showed a multilayered tissue could be described more simply by the homogeneous model if the optical properties of the different layers are not greatly dissimilar 1113 We also intend to test the accuracy of the fit method used here and calculate its associated error.

\section{REFERENCES}

[1] Y.-C. C. et al, "Spatially and angularly resolved spectroscopy for in-situ estimation of concentration and particle size in colloidal suspensions," Analytical and bioanalytical chemistry. , 2016.

[2] A. Ishimaru, Wave propagation and scattering in random media, Academic Press, New York, 1978.

[3] A. C. M. Sakami and V. L. Dez, "Radiative heat transfer in three-dimensional enclosures of complex geometry by using the discrete-ordinates method," J. Quant. Spectrosc. Radiat. Transfer 59, pp. 117-136, 1998.

[4] S. T. Thynell, "Discrete-ordinates method in radiative heat transfer," International Journal of Engineering Science 36, pp. 1651-1675, 1998.

[5] N. M. Subhash C. Mishra, Hillol K. Roy, "Discrete ordinate method with a new and a simple quadrature scheme," J. Quant. Spectrosc. Radiat. Transfer 101, p. 249262, 2006.

[6] C. Larry Reynolds and A.Ishimaru, "Diffuse reflectance from a finite blood medium: application to the modeling of fiber optic catheters," Applied optics 15, pp. 2059-2067, September 1976.

[7] C. Maetzler, "Matlab functions for mie scattering and absorption," Institute of Applied Physics, University of Bern, 2002.

[8] D. R. H. Craig F. Bohren, Absorption and Scattering of light by small particles, WILEY-VCH, 2004.

[9] M. Newville, T. Stensitzki, D. B. Allen, and A. Ingargiola, "LMFIT: Non-Linear Least-Square Minimization and Curve-Fitting for Python," Sept. 2014.

[10] H. R.A.J.Groenhuis and J. Bosch, "Scattering and absorption of turbid materials determined from reflection measurements. 1: Theory," Applied optics 22, pp. 2456-2462, 15 August 1983.

[11] H. R.A.J.Groenhuis and J. Bosch, "Scattering and absorption of turbid materials determined from reflection measurements. 2: Measuring method and calibration," Applied optics 22, pp. 2463-2467, 15 August 1983.

[12] W.G.Egan and T.W.Hilgeman, Optical Properties of Inhomogeneous Materials, Academic, 1979.

[13] E. J.M.Schmitt, G.X.Zhou and R.T.Wall, "Multilayer model of photon diffion in skin," J. Opt. Soc. Am 7, pp. 2141-2153, 1990. 